\title{
Immunotherapies in the management of epidermal growth factor receptor mutated non-small cell lung cancer: a role will be found?
}

\author{
Francesca Casaluce, Cesare Gridelli \\ Division of Medical Oncology, S.G. Moscati Hospital, Avellino, Italy \\ Correspondence to: Cesare Gridelli. Division of Medical Oncology, S.G. Moscati Hospital, Avellino, Italy. Email: cgridelli@libero.it. \\ Comment on: Lisberg A, Cummings A, Goldman JW, et al. A Phase II Study of Pembrolizumab in EGFR-Mutant, PD-L1+, Tyrosine Kinase Inhibitor \\ Naive Patients With Advanced NSCLC. J Thorac Oncol 2018;13:1138-45.
}

Submitted Sep 14, 2018. Accepted for publication Sep 25, 2018.

doi: $10.21037 /$ tlcr.2018.09.18

View this article at: http://dx.doi.org/10.21037/tlcr.2018.09.18

Immunotherapies have been incorporated in the treatment strategy of advanced non-small cell lung cancer (NSCLC), with worldwide pembrolizumab approval as upfront single agent for tumors with programmed cell death-ligand 1 (PD-L1) tumor proportion score (TPS) of $\geq 50 \%$, and recently with the US Food and Drug Administration (FDA) and the European Medicines Agency (EMA) approvals of pembrolizumab in combination with chemotherapy for non-squamous lung cancer, irrespective of PD-L1 status. On other hand, targeted tyrosine-kinase inhibitors (TKIs) represent the best first-line choice for patients whose tumors have sensitizing epidermal growth factor receptor (EGFR) mutations. Although the presence or not of these predictive biomarkers play a crucial role for physicians' choice, no final consensus has been established regarding the optimal treatment sequencing for EGFR mutant and PD-L1 positive NSCLC patients.

To date, subgroup analyses from phase III pivotal trials showed pretreated patients with sensitizing EGFR mutations as poor responders to PD-L1 blockade therapies (1-4). A recent meta-analysis on the efficacy of immune checkpoint inhibitors versus docetaxel in pretreated advanced NSCLC patients has been reported by Lee and colleagues (5). The analysis of over 3,000 patients enrolled in five landmark trials confirmed a significantly survival improvement with immuno-agents than docetaxel as second-line for all population, with a $32 \%$ reduction in risk of death. However, according to EGFR mutational status, the survival improvement was confirmed only for EGFR wild-type patients [overall survival (OS) hazard ratio (HR): 0.67; $\mathrm{P}<0.001$ ], but not in those EGFR mutated (OS HR:
$1.11 ; \mathrm{P}=0.54)(5)$.

Yet the underlying association between EGFR mutation and immune resistance remains largely unclear. Preclinical and retrospective data suggested the upregulation of PD-1 axis pathway as a typical mechanism of immune evasion in EGFR mutated tumors (6-8). During EGFR blockade, the "immuno-target" PD-L1 seems to disappear, as described in some cell lines studies treated with erlotinib, conflicting with PD-L1 upregulation during treatment with gefitinib, as clinically reported in serial biopsy specimens $(8,9)$. The circumstantial nature of those data, the conflicting results on PD-L1 fluctuations during EGFR blockade and post-progression, the lack of knowledge about EGFR heterogeneous cohorts analyzed in clinical trials (such as the specific impact of the type of EGFR mutations and of pretreatment on immuno-treatment efficacy-and the exclusion of EGFR-mutated patients from two pivotal PD-1 first-line trials $(10,11)$, does not clarify whether PD-1 axis inhibition might work in untreated EGFR positives patients. In order to add the rapid responses of EGFR TKI with durable disease control with immunotherapy, the combination approach was firstly valuated in a small sample size of EGFR TKI naïve patients treated in early phase trials, reporting an enhanced activity, but unexpectedly high toxicity rates (12-14).

Subsequently, based on BRAF mutant melanoma results reporting immunotherapy alone prior targeted TKI-directed therapy as validated approach (15), the current study reported by Lisberg and colleagues, examined the activity and safety of immunotherapy if administered before an EGFR TKI in the sequencing treatment plan 
for EGFR mutant NSCLC patients (16). This open-label, single cohort, uncontrolled, phase II trial was designed to show a response rate to pembrolizumab of $26 \%$ or more [overall response rate (ORR) as primary endpoint], substantially lower than that historically observed with TKIs. They report on a small cohort of 11 untreated advanced NSCLC patients with EGFR mutated and PD-L1 expression levels of at least $1 \%$, receiving pembrolizumab as fist-line (dose of $200 \mathrm{mg}$ intravenously every three weeks). At failure of immunotherapy, patients were treated with EGFR TKI, evaluating efficacy and safety of the subsequent targeted treatment as secondary endpoints. Among patients studied, only one responded to pembrolizumab, with surprising EGFR wild type status at repeated analysis of original tumor specimen. No responses to pembrolizumab were reported for remaining 10 patients, with stable disease as best response for 7 patients. Notably, pembrolizumab as fist-line failed even among 7 patients EGFR mutant with strongly positivity of PD-L1 status $(\geq 50 \%)$. This trial seems to confirm immunotherapy alone as unappropriated firstline treatment choice for EGFR mutated, suggesting that checkpoint inhibitors should be considered only after other effective therapies have been exhausted for those setting of patients. To note, caution regarding definitive conclusion on safety data is mandatory. Administering pembrolizumab before EGFR TKI seems to not increase rates of treatment related toxicities, despite one patient reported adrenal insufficiency due to pembrolizumab, with a subsequent worse impact on quality of life, clearly avoided if standard EGFR TKI treatment would be used. The contribution of prior pembrolizumab to the fatal pneumonitis on erlotinib reported in one Caucasian patient is highly supposed and under valuation, considering the low incidence of this toxicity described in literature (17). On other hand, the efficacy of TKI seems to be not affected by preceding PD-1 axis inhibition, with $71 \%$ of patients $(5 / 7)$ still in treatment with erlotinib as second-line, but with unclear durability, considering less than four months as median duration of TKI administration. If this study seems to confirmed the failure of immunotherapy alone for EGFR mutant patients, a new promising role is recently suggested from phase III IMpower150 trial. The use of a four-drug regimen as first-line (the anti-vascular endothelial growth factor (VEGF) bevacizumab plus the anti-PD-L1 atezolizumab plus chemotherapy with carboplatin and paclitaxel) in 108 patients with EGFR/anaplastic lymphoma kinase (ALK) genomic alterations reported, in a subgroup analysis, significant survival outcome improvement, reducing of $46 \%$ the risk of death (18).

Ongoing researches would clarify which mechanisms underlying for a poor response to immune checkpoint blockade in EGFR mutant tumors, with new emerging suggestions. The presence of uninflamed tumor microenvironment and the low mutational load are both highly correlated with NSCLC harboring EGFR mutations, potentially explaining the impaired response to immunotherapies: the uninflamed phenotype resulted in the immunological tolerance, as well as low nonsynonymous mutation burden is emerging related to worse clinical outcomes with immunotherapies (19). The research of other mechanisms clarifying the weak immunogenicity of EGFR mutant, as well as of new predictive biomarkers for better selection of patients potentially suitable of immunotherapy will be mandatory. For selecting EGFR mutated patients for immunotherapy, the knowledge of tumor mutation burden and PD-L1 expression status may not be enough, requiring other helpful details, such as the time to progression at first-line TKIs and the mechanism of secondary mutations. Notably, the PD-L1 expression seems to correlate with EGFR T790M mutation status (biomarker of resistance to anti-EGFR TKIs), with higher levels in those negative samples (20). Recently, a clinical case suggested that shorter progression free survival at first-line EGFR-TKI treatment for EGFR mutant/high PD-L1 NSCLC patient, and with acquired resistance T790M negative, could encourage physician to try second-line immunotherapy (21). In conclusion, the way of immunotherapy for EGFR mutated patients seems to be unlikely way to go, but not yet definitively stopped.

\section{Acknowledgement}

None.

\section{Footnote}

Conflicts of Interest: Dr. Gridelli received honoraria as speaker bureau and advisory board member from Astra Zeneca, BMS, MSD and Roche. The other author has no conflicts of interest to declare.

\section{References}

1. Borghaei H, Paz-Arez L, Horn L, et al. Nivolumab versus Docetaxel in Advanced Non squamous Non-Small Cell Lung Cancer. N Engl J Med 2015;373:1627-39. 
2. Gainor JF, Shaw AT, Sequist LV, et al. EGFR Mutations and ALK Rearrangements Are Associated with Low Response Rates to PD-1 Pathway Blockade in Non-Small Cell Lung Cancer (NSCLC): A Retrospective Analysis. Clin Cancer Res 2016;22:4585-93.

3. Garon EB, Rizvi NA, Hui R, et al. Pembrolizumab for the Treatment of Non-Small-Cell Lung Cancer. New Engl J Med 2015;372:2018-28.

4. Hellmann MD, Garon EB, Gandhi L, et al. MINI03.05 Efficacy of Pembrolizumab in Key Subgroups of Patients with Advanced NSCLC. J Thorac Oncol 2015;10:S261-406.

5. Lee CK, Man J, Lord S, et al. Clinical and Molecular Characteristics Associated With Survival Among Patients Treated With Checkpoint Inhibitors for Advanced NonSmall Cell Lung Carcinoma. A Systematic Review and Meta-analysis. JAMA Oncol 2018;4:210-6.

6. Akbay EA, Koyama S, Carretero J, et al. Activation of the PD-1 pathway contributes to immune escape in EGFRdriven lung tumors. Cancer Discov 2013;3:1355-63.

7. Zhang $\mathrm{N}$, Zeng $\mathrm{Y}$, Du W, et al. The EGFR pathway is involved in the regulation of $\mathrm{PD}-\mathrm{L} 1$ expression via the IL-6/JAK/STAT3 signaling pathway in EGFR-mutated non-small cell lung cancer. Int J Oncol 2016;49:1360-8.

8. Azuma K, Ota K, Kawahara A, et al. Association of PD-1 overexpression with activating EGFR mutations in surgically resected non-small cell lung cancer. Ann Oncol 2014;25:1935-40.

9. Han JJ, Kim DW, Koh J, et al. Change in PD-L1 expression after acquiring resistance to gefitinib in EGFRmutant non-small-cell lung cancer. Clin Lung Cancer 2016;17:263-70.e2

10. Reck M, Rodríguez-Abreu D, Robinson AG, et al. Pembrolizumab versus Chemotherapy for PD-L1Positive Non-Small-Cell Lung Cancer. N Engl J Med 2016;375:1823-33.

11. Carbone DP, Reck M, Paz-Ares L, et al. First-Line Nivolumab in Stage IV or Recurrent Non-Small-Cell Lung Cancer. N Engl J Med 2017;376:2415-26.

Cite this article as: Casaluce F, Gridelli C. Immunotherapies in the management of epidermal growth factor receptor mutated non-small cell lung cancer: a role will be found? Transl Lung Cancer Res 2018;7(Suppl 4):S370-S372. doi: 10.21037/ tlcr.2018.09.18
12. Gibbons DL, Chow LQ, Kim DW, et al. Efficacy, safety and tolerability of MED (durvalumab) a human IgG1 antiprogrammed cell death-ligand-1 antibody, combined with gefitinib: a phase I expansion in TKI-naïve patients with EGFR mutant NSCLC. J Thorac Oncol 2016;11:S79.

13. Ma BB, Rudin CM, Cervantes A, et al. Preliminary safety and clinical activity of erlotinib plus atezolizumab from a phase $1 \mathrm{~b}$ study in advanced NSCLC. Ann Oncol 2016;27:ix139-56.

14. Ahn MJ, Yang J, Yu H, et al. 136O: Osimertinib combined with durvalumab in EGFR-mutant non-small cell lung cancer: results from the TATTON phase Ib trial. J Thorac Oncol 2016;11:S115.

15. Jang S, Atkins MB. Which drug, and when, for patients with BRAF-mutant melanoma? Lancet Oncol 2013;14:e60-9.

16. Lisberg A, Cummings A, Goldman JW, et al. A Phase II Study of Pembrolizumab in EGFR-mutant, PD-L1+, Tyrosine Kinase Inhibitor (TKI) Naïve Patients with Advanced NSCLC. J Thorac Oncol 2018;13:1138-45.

17. Shepherd FA, Rodrigues Pereira J, Ciuleanu T, et al. Erlotinib in previously treated non-small-cell lung cancer. N Engl J Med 2005;353:123-32.

18. Socinsky MA, Jotte RM, Cappuzzo F, et al. Atezolizumab for First-Line Treatment of Metastatic Nonsquamous NSCLC. N Engl J Med 2018;378:2288-301.

19. Dong ZY, Zhang JT, Liu SY, et al. EGFR mutation correlates with uninflamed phenotype and weak immunogenicity, causing impaired response to PD-1 blockade in non-small cell lung cancer. Oncoimmunology 2017;6:e1356145.

20. Hata A, Katakami N, Nanjo S, et al. Programmed deathligand 1 expression and T790 M status in EGFR-mutant non-small cell lung cancer. Lung Cancer 2017;111:182-9.

21. Kunimasa K, Nakamura H, Sakai K, et al. Heterogeneity of EGFR-mutant clones and PD-L1 highly expressing clones affects treatment efficacy of EGFR-TKI and PD-1 inhibitor. Ann Oncol 2018. [Epub ahead of print]. 